\title{
Statistical 3D Vessel Segmentation Using a Rician Distribution
}

\author{
Albert C. S. Chung and J. Alison Noble \\ Department of Engineering Science, Oxford University, Oxford, OX1 3PJ. \\ \{albert, noble\} Orobots.ox.ac.uk
}

\begin{abstract}
This paper presents an extended version of the fully automated 3D cerebral vessel reconstruction algorithm developed by Wilson and Noble [11] which is applicable to time-of-flight (TOF) and phase contrast (PC) magnetic resonance angiography (MRA) images. We introduce a Rician distribution for background noise modelling and use a modified EM (Expectation-Maximization) algorithm for the parameter estimation procedure. The proposed algorithm is applied to PC-MRA images. It is shown that the estimated Rician distribution gives a better quality-of-fit to the observed background noise distribution than a Gaussian distribution. In the experiments reported, the segmented 3D vasculature is shown to be qualitatively comparable with the results obtained from higher resolution TOF MRA images.
\end{abstract}

Keywords: PC/TOF MRA, Vessel Segmentation, EM Algorithm, GDC (Guglielmi Detachable Coil) Treatment, Endovascular Treatment of Aneurysms, Neuorology.

\section{Introduction}

Three dimensional vascular segmentation is an essential prior step for brain aneurysm charaterization which has been shown, for example, to be extremely useful for pre-GDC (Guglielmi detachable coil) treatment planning [12]. Pretreatment vessel examination is commonly performed using magnetic resonance angiography (MRA) because the image intensity is flow related and MRA does not require the use of contrast agent as for example X-ray analysis does. Two typical and major groups of MRA techniques are phase contrast (PC) and timeof-flight (TOF). The main advantage of PC-MRA over TOF-MRA is that it not only gives information about vascular morphology but also provides, additionally, directional-flow images in each orthogonal direction. This work is entirely driven by the growing need of PC-MRA image segmentation. This paper extends the fully automated 3D cerebral vessel reconstruction algorithm developed by Wilson and Noble [11] from TOF-MRA to PC-MRA. PC-MRA is employed for capturing the flow rate (both magnitude and directions) information spatially within a region of interest (ROI) at a particular time. Similar to TOF-MRA, the voxel intensity is flow-encoded [8]. Intensity and flow rate are positively correlated in PC-MRA. However, unlike TOF-MRA, which shows detail anatomical 
structures, a PC-MRA image displays two major and high contrast voxel types: vessel and background. It is common to assume that the background intensities are Gaussian distributed. However, in this paper, we adopt a Rician distribution as the statistical representation of the background intensities because, theoretically, the background intensities follow a Rician distribution $[4,1,9]$. The experiments we present show that the Rician distribution gives a better quality-of-fit than a Gaussian distribution. We present a method for estimating parameters based on modified EM (Expectation-Maximization) algorithm [2]. The proposed algorithm is tested on PC-MRA images and segmentation results are shown.

\section{The Algorithm}

This section briefly explains the statistical models of each voxel type based on the image formation process and physical characteristics of blood flow. These models are combined additively, and their parameters are estimated via a modified EM algorithm. Segmentation criteria are then described.

\subsection{Derivation of the Background Noise and Vessel Models}

We assume the following background noise model to describe the intensity characteristics of the voxels having approximately zero flow rate, i.e. air background or tissues with stationary flow rate. For PC-MRA, the flow-rate of each voxel is encoded separately in three orthogonal directions. The three orthogonal velocity components, $v_{x}, v_{y}$ and $v_{z}$, are then combined to obtain the flow speed $v=\sqrt{v_{x}^{2}+v_{y}^{2}+v_{z}^{2}}$. These velocity components are complex numbers. Each component is computed by the difference between positive and negative gradient Fourier transformed images along the component direction $[1,7]$. We assume that the real and imaginary parts of these complex components are independently Gaussian distributed with different means $\mu_{k}, k=1 \ldots 6$, and the same variance $\sigma^{2}$. The probability density function (p.d.f.) of the flow $v$ is then governed by a Rician distribution, which describes the distribution of the square root of the squared-sum of Gaussian random variables. Since the voxel intensity $i$ and flow $v$ are linearly related, the p.d.f. of the background voxel intensity $f_{b}(i)$ is also Rician distributed and given by,

$$
f_{b}(i)=\frac{i}{\sigma^{2}}\left(\frac{i}{A}\right)^{\frac{N-2}{N}} e^{\frac{-\left(i^{2}+A^{2}\right)}{2 \sigma^{2}}} I_{\frac{N}{2}-1}\left(\frac{i A}{\sigma^{2}}\right) u(i),
$$

where $A^{2}=\sum_{k=1}^{N} \mu_{k}, I$ is the modified Bessel function of real order, $u$ is the unit step function and $N$ is the number of independent Gaussian random variables. $N=6$ for the rest of the paper. A plot of Rician distributions with difference Signal-to-Noise (SNR) ratios $\frac{A}{\sigma}=0.001,1,2 \ldots 5$ and their corresponding skewness measures $\gamma$ are shown in Figure 1a. It shows that a Rician distribution with small SNR tends to be positively skewed and that with large SNR it is symmetric and approximately Gaussian. 


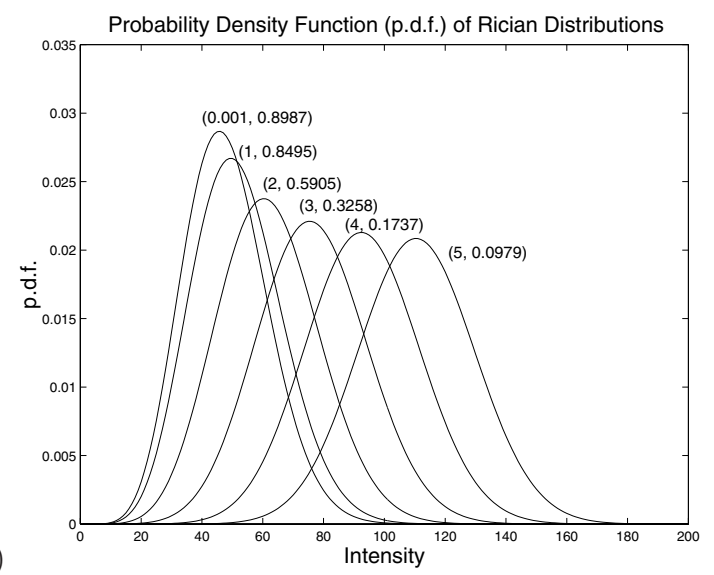

(b)

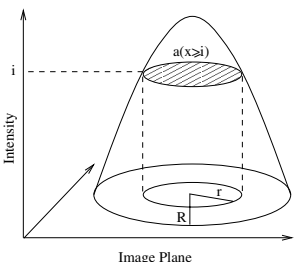

Fig. 1. (a) A plot of Rician distributions with different SNR and their corresponding skewness measures $\left(\frac{A}{\sigma}, \gamma\right)$. This shows that a Rician distribution with small SNR tends to be positively skewed and that with large SNR it is symmetric and approximately Gaussian. (b) A typical laminar flow pattern. $a(x \geq i)$ is the area having intensity $x$ larger than $i$. The probability that a voxel has intensity equal to $i$ is then given by $\left|\frac{d a(x \geq i)}{d i}\right|$.

We assume a vessel model, in which the intensity characteristics of the vessel voxels are assumed to exhibit a laminar flow pattern. The velocity profile across the circular vessel cross-section is then parabolic [3], as shown in Figure 1b. Hence, the intensity profile is $i=C\left(1-\frac{r^{2}}{R^{2}}\right)$, where $i$ is the intensity, $C$ is a constant, $R$ is the vessel radius and $r$ is the distance from vessel centre to the boundary. The p.d.f. $f_{v}(i)$ for a vessel voxel that has intensity $i$ is directly proportional to the area $a(x=i) . f_{v}(i)$ is calculated as the rate-of-change of area having intensity greater than or equal to $i$, i.e. $f_{v}(i) \propto\left|\frac{d a(x \geq i)}{d i}\right|$. The area $a(x \geq i)$ is given by $\pi R^{2}\left(1-\frac{i}{C}\right)$. Therefore, the p.d.f. $f_{v}$ is constant and can be regarded as a uniform distribution, $f_{v}(i)=\frac{1}{i_{\max }}$, where $i_{\max }$ is the maximum intensity in the frequency histogram. Although, in practice, the vessel voxel intensity mainly spreads over the high intensity region the number of vessel voxels is only a small proportion (1\%) of the frequency histogram. Hence, for the sake of simplicity, we assume that the uniform distribution spreads over the entire intensity range $\left(0 \ldots i_{\max }\right)$.

The background noise and vessel models can be combined into a mixture model. The modified EM algorithm [2] can be used to estimate the parameters for the mixture $f(i)=w_{b} f_{b}(i \mid b)+w_{v} f_{v}(i \mid v)$, where $f_{b}(i \mid b)$ and $f_{v}(i \mid v)$ are the conditional probabilities that a voxel has intensity $i$ given that it is a background noise and vessel voxel respectively, and $w_{b}$ and $w_{v}$ are the prior probabilities of a background noise and vessel voxel respectively. In other words, $w_{b}$ and $w_{v}$ are 
the weights of the background and vessel models respectively, and $w_{b}+w_{v}=1$. A procedure for estimating the model parameters is outlined in the next subsection.

\subsection{Parameter Estimation Procedure}

This subsection concerns parameter estimation of a mixture density. Given a mixture model of a Rician and uniform distributions, there are four unknown parameters: $w_{b}, w_{v}, A$ and $\sigma^{2}$ that need to be estimated. The modified EM algorithm is an iterative procedure that can be used to estimate the parameters which maximizes the log-likelihood of the mixture distribution in each iteration [2]. The iterative procedure terminates when the change in log-likelihood or the parameters is sufficiently small.

Let the log-likelihood function be $L=\sum_{i=0}^{i_{\max }} h(i) \log f(i)$, where $h(i)$ is the frequency histogram of the observations and $f(i)$ is the mixture p.d.f.. The change in log-likelihood function is given by

$$
L^{k+1}-L^{k}=\sum_{i=0}^{i_{\max }} h(i) \log \left(\frac{f^{k+1}(i)}{f^{k}(i)}\right)
$$

where index $k$ represents the $k^{\text {th }}$ iteration step. We aim to maximize the change of $\log$-likelihood $L^{k+1}-L^{k}$ until the change is sufficiently small. Suppose that, given an intensity $i$, the posterior probabilities that a voxel belongs to the background noise and vessel are $p(b \mid i)$ and $p(v \mid i)$ respectively. Then $p(b \mid i)=w_{b} f_{b}(i \mid b) / f(i)$. The same applies to $p(v \mid i)$. Also $p(b \mid i)+p(v \mid i)=1$. Equation (2) can be rewritten as

$$
L^{k+1}-L^{k}=\sum_{i=0}^{i_{\max }} h(i) \log \left(p^{k}(b \mid i) \frac{w_{b}^{k+1} f_{b}^{k+1}(i \mid b)}{f^{k}(i) p^{k}(b \mid i)}+p^{k}(v \mid i) \frac{w_{v}^{k+1} f_{v}^{k+1}(i \mid v)}{f^{k}(i) p^{k}(v \mid i)}\right)
$$

by expanding $f^{k+1}(i)$ and multiplying $p^{k}(b \mid i)$ and $p^{k}(v \mid i)$ to both numerator and denominator. By Jensen's inequality, $\log \left(\lambda_{1} x_{1}+\lambda_{2} x_{2}\right) \geq \lambda_{1} \log x_{1}+\lambda_{2} \log x_{2}$ and $\lambda_{1}+\lambda_{2}=1$. Let $\lambda_{1}=p^{k}(b \mid i)$ and $\lambda_{2}=p^{k}(v \mid i)$. Then from Equation (3), we have

$$
L^{k+1}-L^{k} \geq \sum_{i=0}^{i_{\max }} h(i)\left(p^{k}(b \mid i) \log \frac{w_{b}^{k+1} f_{b}^{k+1}(i \mid b)}{f^{k}(i) p^{k}(b \mid i)}+p^{k}(v \mid i) \log \frac{w_{v}^{k+1} f_{v}^{k+1}(i \mid v)}{f^{k}(i) p^{k}(v \mid i)}\right)(4)
$$

Therefore, maximizing the right-hand-size of the inequality in Equation (4) is equivalent to maximizing the change in log-likelihood until the log-likelihood converges to a stationary point.

Maximization with respect to $w_{b}^{k+1}$ and $w_{v}^{k+1}$ : the right-hand-side of Equation (4) can be rearranged to isolate the terms related to $w_{b}^{k+1}$ and $w_{v}^{k+1}$. Let

$$
Q_{w}=\sum_{i=0}^{i_{\max }} h(i)\left(p^{k}(b \mid i) \log w_{b}^{k+1}+p^{k}(v \mid i) \log w_{v}^{k+1}\right) .
$$


Then we need to maximize $Q_{w}$ under that constrain $w_{b}^{k+1}+w_{v}^{k+1}=1$, i.e. maximize $Q_{w}+\lambda\left(1-w_{b}^{k+1}-w_{v}^{k+1}\right)$, where $\lambda$ is the lagrange multiplier. Setting the derivatives with respect to $w_{b}^{k+1}$ and $w_{v}^{k+1}$ to zero gives,

$$
\lambda w_{b}^{k+1}=\sum_{i=0}^{i_{\max }} h(i) p^{k}(b \mid i) \text { and } \lambda w_{v}^{k+1}=\sum_{i=0}^{i_{\max }} h(i) p^{k}(v \mid i)
$$

Summing Equations (6) gives $\lambda=M$, which $M$ is the total number of voxels. Hence, we obtain

$$
w_{b}^{k+1}=\frac{1}{M} \sum_{i=0}^{i_{\max }} h(i) p^{k}(b \mid i) \text { and } w_{v}^{k+1}=\frac{1}{M} \sum_{i=0}^{i_{\max }} h(i) p^{k}(v \mid i) .
$$

The initial values of $w_{b}^{0}$ and $w_{v}^{0}$ we have used in our experiments are 0.99 and 0.01 respectively.

Maximization with respect to $A_{k+1}$ and $\sigma_{k+1}^{2}$ : the right-hand-side of Equation (4) can be rearranged to isolate the terms related to $A_{k+1}$ and $\sigma_{k+1}^{2}$.

$$
Q_{A, \sigma^{2}}=\sum_{i=0}^{i_{\max }} h(i) p^{k}(b \mid i) \log f_{b}^{k+1}(i \mid b) .
$$

Setting the derivatives of $Q_{A, \sigma^{2}}$ with respect to $A_{k+1}$ and $\sigma_{k+1}^{2}$ to zero gives

$$
\begin{gathered}
\frac{\partial Q_{A, \sigma^{2}}}{\partial A_{k+1}}=0 \Rightarrow \sum_{i=0}^{i_{\max }} h(i) p^{k}(b \mid i)\left[\left(1-\frac{N}{2}\right) \frac{1}{A_{k+1}}-\frac{A_{k+1}}{\sigma_{k}^{2}}+\frac{I_{v}^{\prime}}{I_{v}} \frac{i}{\sigma_{k}^{2}}\right]=0, \\
\frac{\partial Q_{A, \sigma^{2}}}{\partial \sigma_{k+1}^{2}}=0 \Rightarrow \sum_{i=0}^{i_{\max }} h(i) p^{k}(b \mid i)\left[\frac{i^{2}+A_{k}^{2}-2 \sigma_{k+1}^{2}}{2 \sigma_{k+1}^{4}}-\frac{I_{v}^{\prime}}{I_{v}} \frac{i A_{k}}{\sigma_{k+1}^{4}}\right]=0
\end{gathered}
$$

where $v=\frac{N}{2}-1, I_{v}=I_{v}(y), I_{v}^{\prime}=\frac{d I_{v}}{d y}$. For Equation (9), $y=\frac{i A_{k+1}}{\sigma_{k}^{2}}$. For Equation (10), $y=\frac{i A_{k}}{\sigma_{k+1}^{2}}$.

Figures $2 \mathrm{a}$ and $2 \mathrm{~b}$ show that the curves of derivatives of $Q_{A, \sigma^{2}}$ with respect to $A_{k+1}$ and $\sigma_{k+1}^{2}$ versus $A_{k+1}$ and $\sigma_{k+1}^{2}$ are monotonic decreasing, which implies that the curves of $Q_{A, \sigma^{2}}$ with varying $A_{k+1}$ and $\sigma_{k+1}^{2}$ are convex and have a unique maximum. Hence, the solutions $A_{k+1}$ and $\sigma_{k+1}^{2}$ of Equations (9) and (10) will maximize the value of $Q_{A, \sigma^{2}}$. Equations (9) and (10) can be solved numerically to find $A_{k+1}$ and $\sigma_{k+1}^{2}$ using, for example, the Newton-Raphson method. As shown in Figures $2 \mathrm{a}$ and $2 \mathrm{~b}$, both curves are approximately linear for a wide range of $A_{k+1}$ and $\sigma_{k+1}^{2}$. Given the initial guesses of $A_{k}$ and $\sigma_{k}^{2}$, the NewtonRaphson method converged after approximately 2 iterations in practice. The initial values of $A_{0}$ and $\sigma_{0}^{2}$ we have used in our experiments are $\sqrt{E\left[i^{2}\right]-N \sigma^{2}}$ and $\sigma^{2}$ the histogram variance respectively, where $E\left[i^{2}\right]$ is the expected value 
(a)

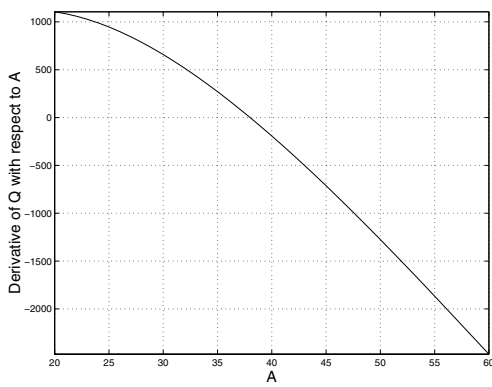

(b)

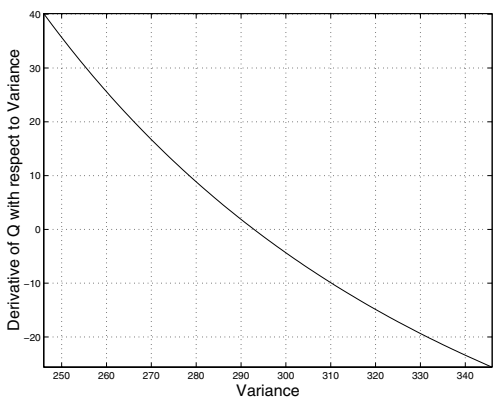

Fig. 2. (a) and (b) show that the curves of derivatives versus $A_{k+1}$ and $\sigma_{k+1}^{2}$ are monotonic decreasing, which implies that the curves of $Q_{A, \sigma^{2}}$ with varying $A_{k+1}$ and $\sigma_{k+1}^{2}$ are convex and have a unique maximum.

of $i^{2}$. The termination criterion for Newton-Raphson method was found empirically. The termination conditions in the Newton-Raphson method were set to be $\left|A_{k+1}-A_{k}\right| \leq 0.1$ and $\left|\sigma_{k+1}^{2}-\sigma_{k}^{2}\right| \leq 5$. These choices were found to keep the change in $Q_{A, \sigma^{2}}$ approximately $0.5 \%$ per iteration of the EM algorithm. The EM algorithm applies the same termination criteria and converged after approximately 15 iterations.

\subsection{Results}

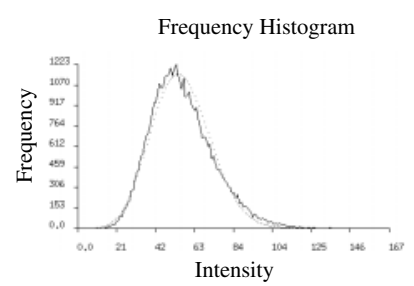

(a)

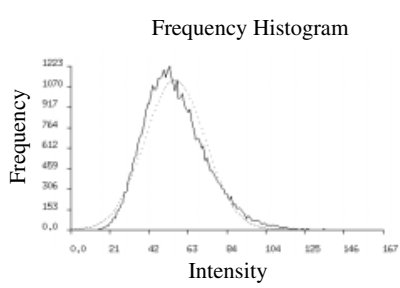

(b)

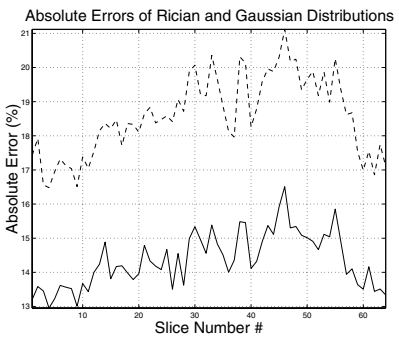

(c)

Fig. 3. (a) and (b) show the estimation results when $f_{b}$ is assumed to be Rician and Gaussian respectively. The solid line is the observed histogram and the dotted line is the estimated histogram. For 64 slices of a PC MRA volume, (c) shows the absolute errors (\%) of the Rician (solid) and Gaussian (dashed) distributions versus the slice number. This shows that the Rician distribution gives a consistent better quality-of-fit for the background noise distribution than a Gaussian distribution. 
Figure $3 \mathrm{a}$ and $3 \mathrm{~b}$ shows a typical EM estimation result when the background voxel intensity $f_{b}$ is assumed to be Rician and Gaussian respectively. Estimations were performed in each of the 64 slices of a PC-MRA volume. Figure 3c displays the absolute errors of the Rician (solid) and Gaussian (dashed) distributions versus slice number. Absolute error is defined as the absolute difference between the observed and estimated histograms $\sum_{i=0}^{i_{\max }} \frac{1}{M}\left|h_{\text {observed }}(i)-h_{\text {estimated }}(i)\right| \times$ $100 \%$. Note that the Rician distribution consistently gives a better quality-of-fit (about 5\%) for the background noise distribution than a Gaussian distribution. This is because of the low signal-to-noise ratio (SNR) of the background. Low SNR magnifies the contributions of Gaussian noise in each orthogonal component to the background signal. Therefore, the background distribution is corrupted from a Gaussian to a Rician distribution. A Gaussian distribution is still a good approximation when the SNR is significantly large $\left(\frac{A}{\sigma} \geq 3\right)[4]$.

\subsection{Segmentation Criteria}

Given an estimated mixture model, a volume of PC-MRA can be segmented statistically on the basis of the MAP (Maximum-A-Posterior) criterion, which is conceptually different from the criteria of feature-based methods demanding the extraction of relevant spatial features, e.g. edge, curvature [5,6] or intensity variance, or velocity coherence among neighbouring voxels [10], as the criteria for vessel segmentation. Using MAP, a voxel is classified as a vessel voxel when the vessel probability $w_{v} f_{v}(i \mid v)$ is greater than the background probability $w_{b} f_{b}(i \mid b)$. Therefore, a threshold $i_{t}$ can be found by the intersection of the two probability distributions.

\section{Frequency Histogram}

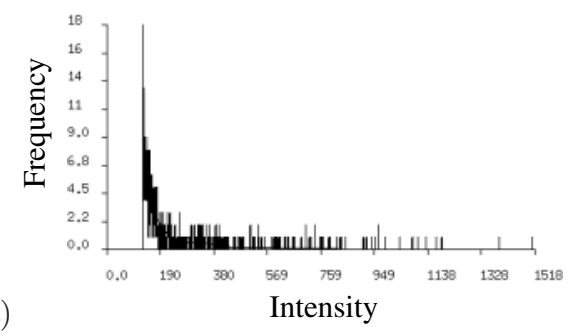

(b)

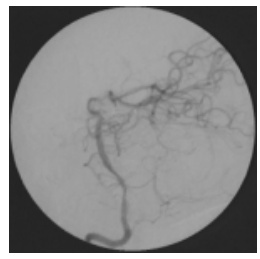

Fig. 4. (a) shows that the histogram frequency increases as the intensity decreases. This is because the number of relatively slow flow (low intensity) small vessel voxels is greater than that of fast flow large vessel voxels. As shown in (b), which is an X-ray image of the same brain, most of the small subsidiary vessels lead from a single major vessel. The threshold is found by finding the point at the maximum planar curvature. In (a), the maximum curvature is at intensity value 198 . 
The threshold $i_{t}$ cuts the observed histogram into two portions. The high intensity portion describes the intensity distribution of the vessel voxels, as shown in Figure 4a. Note that the high intensity portion of the histogram follows the uniform distribution and is consistent with the vessel model. However, note also that the histogram frequency increases as the intensity decreases. This is because the number of relatively slow-flow (low intensity) small-vessel voxels is greater than that of fast-flow large-vessel voxels. As shown in Figure 4b, most of the small subsidiary vessels lead from the single major vessel. Therefore, when the intensity decreases, the number of voxels increases because of the increase in the number of low-intensity small vessels, which in turn causes an increase in the histogram frequency. This change in frequency can be found by finding the point $i_{t}^{\prime}$ at the maximum planar curvature of the smoothed curve. Curvature is defined as $\frac{d^{2} h(i)}{d i^{2}} /\left[1+\left(\frac{d h(i)}{d i}\right)^{2}\right]^{\frac{3}{2}}$. In Figure $4 \mathrm{a}, i_{t}$ and $i_{t}^{\prime}$ are approximately 120 and 198 respectively.

Figure 5a shows a sub-image from a PC-MRA slice. Figure 5b shows the segmentation results using the threshold $i_{t}^{\prime}$. In this figure voxels with intensity greater than $i_{t}^{\prime}$ are labelled white. Figure $5 \mathrm{c}$ shows the segmentation results using the threshold $i_{t}$. Here voxels with intensity between $i_{t}$ and $i_{t}^{\prime}$ are labelled white; those greater than $i_{t}^{\prime}$ labelled grey. This example illustrates that slow-flow small-vessel voxels are now detected and appear adjacent to the fast flow large vessel voxels. This means the estimated size of major vessels is larger without the correction.

(a)
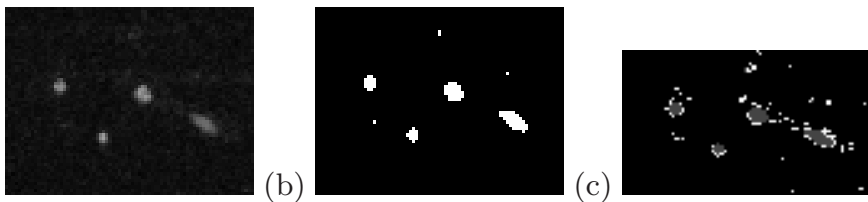

Fig. 5. (a) shows a sub-image from a PC-MRA slice; (b) shows the segmented image using the corrected threshold $I_{t}^{\prime} ;(c)$ shows the segmented image using the threshold $I_{t}$ (found by MAP). Slow flow small vessel voxels are detected and appear adjacent to the fast flow large vessel voxels. This means the size of major vessels is overestimated without the correction.

\section{Results}

Intracranial scans (both PC-MRA and TOF-MRA) of a volunteer (without an aneurysm) were performed using a 1.5 T Siemens Magnetom Vision MR scanner at the Radcliffe Infirmary, Oxford. The volume size was $192 \times 256 x 48$ voxels and voxel size $0.82 \mathrm{~mm} \times 0.78 \mathrm{~mm} \times 1.46 \mathrm{~mm}$. The segmentation algorithm was applied to the whole PC-MRA volume. The segmented 3D vasculature is shown in Figure 6a. A high resolution TOF-MRA image of the same volunteer was also acquired (voxel size $0.67 \mathrm{~mm} \times 0.39 \mathrm{~mm} \times 1.46 \mathrm{~mm}$ and $225 \times 512 \mathrm{x} 48$ voxels). 
The segmentation method [11] was applied to the data. The result is shown in Figure 6b. Observe that although PC-MRA is acquired at a lower scanning resolution, as compared with TOF-MRA, all the major vessels are clearly shown and reconstructed. To verify the aneurysm detection ability of the new algorithm, PC-MRA data from a patient with two small aneurysms was acquired at the same resolution and volume size. Figure $6 \mathrm{c}$ shows a $3 \mathrm{D}$ vascular segmentation of the data. The two aneurysms are clearly detected (pointed by the arrows). The computational time of the current implementation of the algorithm is approximately 10 seconds on a SGI $200 \mathrm{MHz}$ workstation.

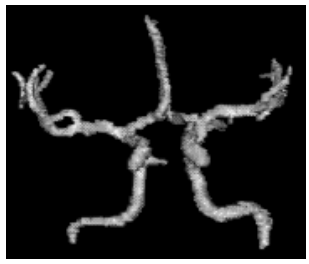

(a) $P C-M R A$

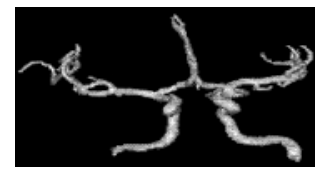

(b) $T O F-M R A$

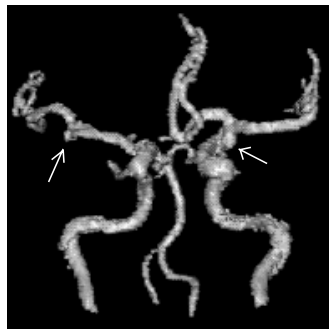

(c) $P C-M R A$

Without an aneurysm Without an aneurysm

Fig. 6. Segmentation Results

\section{Conclusion}

We have presented an extended version of the fully automated 3D cerebral vessel segmentation algorithm [11] applicable to PC-MRA. Conceptually, the proposed algorithm falls into a probabilistic framework, in which the two major voxel types: background and vessel are statistically modelled. Statistical models were derived on the basis of the signal formation process and physical characteristics of the flow pattern. We show that a threshold found by the intersection of the two probabilistic distributions enables a good segmentation of the large major vessels to be performed. The results show that the estimated distribution well approximates the true (observed) background and vessel distributions. The main advantage of PC-MRA over TOF-MRA is that it gives additional directional flow images in the three orthogonal directions. Our next step is to perform directional velocity mapping on the segmented $3 \mathrm{D}$ vasculature such that the flow pattern around the region of aneurysm can be visualized and intensively studied.

\section{Acknowledgements}

We thank Dr. J. Byrne at the Department of Neuroradiology of the Radcliffe Infirmary, Oxford for providing data and helpful discussions. A. Chung is funded by a postgraduate scholarship award from the Croucher Foundation, Hong Kong. 


\section{References}

1. A.H. Andersen and J.E. Kirsch. Analysis of noise in phase contrast MR imaging. Med. Phy., 23(6):857-869,1996. 83

2. C. Bishop. Neural Networks for Pattern Recognition. Clarendon Press,1995. 83, 84,85

3. C.G. Caro,T.J. Pedley et al. The Mechanics of the Circulation. O.U.P.,1978. 84

4. H. Gudbjartsson and S. Patz. The Rician distribution of noisy MRI data. Mag. Reson. Med., 34:910-914,1995. 83, 88

5. K. Krissian,G. Malandain and N. Ayache. Model-based multiscale detection and reconstruction of 3D vessels. Tech. Report RR-3442, INRIA,1998. 88

6. T.McInerney and D.Terzopoulos. Topologically Adaptable Snakes. ICCV'95, 840-5 88

7. N.J. Pelc,M.A. Bernstein et al. Encoding strategies for three-direction Phase Contrast MR imaging of flow. J. Mag. Reson. Imag., 1:405-413,1991. 83

8. P.A. Rinck. Magnetic Resonance in Medicine, Blackwell,1993. 82

9. J. Sijbers,A.J. den Dekker,J.V. Audekerke,M. Verhoye and D. Van Dyck. Estimation of the noise in magnitude MR images. Mag. Reson. Imag., 16(1):87-90,1998. 83

10. P. Summers,A. Bhalerao and D. Hawkes. Multi-resolution, Model-based segmentation of MR Angiograms. J. Mag. Reson. Imag., 7(6):950-957,1997. 88

11. D. Wilson and J. Noble. Segmentation of cerebral vessels and aneurysms from MR angiography data. IPMI, 423-428,1997. 82, 90

12. D.L. Wilson. An Improved Planning Protocol for the Endovascular Treatment of Intracranial Aneurysms, D.Phil.Thesis,U. of Oxford,1998. 82 